\title{
Monte Carlo simulation of branched and crosslinked polymers
}

\author{
Fernando A. Escobedo and Juan J. de Pablo \\ Department of Chemical Engineering, University of Wisconsin-Madison, Madison, Wisconsin 53706-1691
}

(Received 7 August 1995; accepted 28 November 1995)

\begin{abstract}
Novel Monte Carlo simulation techniques are presented for efficient isobaric-isothermal simulations of branched chains and polymer networks with tri- and tetra-functional sites. Molecular rearrangements are performed by means of extended continuum configurational bias moves applied to single-path polymer portions. Volume fluctuations are performed via slab moves, which are extended in this work to effectively handle networks of arbitrary complexity. These methods are applied to determine the volumetric properties of linear and branched chains (with athermal and square-well interaction sites). Novel results are also presented for the compressibility of athermal and thermal polymer networks having a perfect, diamondlike connectivity. () 1996 American Institute of Physics. [S0021-9606(96)50509-1]
\end{abstract}

\section{INTRODUCTION}

Branched and crosslinked polymers are often encountered in elastomers (rubbers), numerous thermoplastic and thermosetting resins, and organic and inorganic gels. These materials find applications as sealants, coatings, and inert or interactive supports, not only in traditional fields (i.e., automotive, textile, and construction industries) but also in more modern biomedical applications (i.e., contact lenses, drug delivery systems, etc.). The mechanical properties of branched or crosslinked structures are largely determined by their topology. Unfortunately, their experimental characterization is difficult; computer simulations therefore provide a valuable tool for understanding these systems at a fundamental level.

While models of linear polymers have been studied extensively, molecular simulations of nonlinear polymers are still at a very early stage of development. The presence of branch-linking sites or nodes introduces additional connectivity constraints which reduce their mobility in phase space, thereby rendering difficult their simulation.

Molecular dynamics (MD) methods, on the one hand, can in principle be used to simulate branched polymers. Hybrid MC methods ${ }^{1}$ can also be used for these systems since molecular rearrangements are performed by an MD-type of procedure. Unfortunately, due to the simplifications needed to render the system's dynamics more tractable, MD methods for hard-core, rigid-bond molecules are necessarily approximate. $^{2,3}$ Another important limitation of MD-related methods is that, in contrast to Monte Carlo (MC) methods, phase equilibria are somewhat difficult to simulate. Conventional MC methods, on the other hand, are inefficient for moving nodal sites and decorrelating successive configurations. Simple whole-particle displacement or rotation moves ${ }^{4,5}$ would not alter an intramolecular configuration, and simple reptation moves ${ }^{6}$ are not applicable to branched chains.

Simulations of branched polymers have been scarce. ${ }^{7}$ To the best of our knowledge, branched chain molecules with attractive interactions have not been investigated before. Available simulation results for athermal systems indicate that linear and branched chain molecules with the same num- ber of total sites have almost identical PV behavior. It is not clear, however, whether a similar trend will be observed with molecules bearing attractive potentials (experimental observations indicate that branching can have important effects on the thermodynamic properties of real polymers).

Gao and Weiner ${ }^{8}$ and Kremer, Grest, and Duering ${ }^{9-11}$ have conducted a number of studies of polymer networks using molecular dynamics. They have investigated several dynamic and structural properties of model elastomers (i.e., calculation of the elastic modulus). Gao and Weiner simulated small-size, highly idealized representations of a polymer network to study segment orientation and chain stresses in stretched networks. ${ }^{8}$ Kremer and co-workers prepared their networks by crosslinking a homopolymer melt with polyfunctional crosslinkers attached to chain ends using molecular dynamics; this approach allowed them to study the kinetics of crosslinking. ${ }^{11}$ These investigations, however, have not addressed the simulation of PVT and phase equilibrium properties of networks.

Although MC methods tend to be significantly more involved than MD methods, they offer greater flexibility; special techniques can be tailored to deal more effectively with specific systems. In this context, continuum configurationalbias MC methods $(\mathrm{CCB})^{12,13}$ are particularly useful for performing molecular rearrangements in chainlike molecules. We have recently proposed extensions of these methods ${ }^{14}$ (ECCB) which permit rearrangement not only of end portions of the chain (as in the original prescription) but also of inner portions, including branch-linking sites.

Another important and often overlooked element of polymer simulation is the algorithm employed to perform volume fluctuations, which plays a key role in the isothermal-isobaric ensemble and in all available phase equilibrium simulation methods. Conventional volume moves entail a change in the size of the simulation box; typically, the internal configuration of the molecules is preserved while their center of mass is rescaled. ${ }^{5}$ Periodic boundary conditions are commonly used around the simulation box to simulate seemingly infinite systems. During a conventional volume move, a crosslinked polymer inside the box can not preserve its internal configuration because res- 
caling its coordinates isotropically leads to overlaps and/or broken bonds. A systematic and effective means for concertedly rearranging the polymer network while changing the volume is necessary to relax the system to low, viable energy states. While force-bias or hybrid techniques could be implemented for this purpose, such global rearrangements, if fruitful, would be inefficient.

Our recently proposed slab moves provide an attractive means of performing volume fluctuations. ${ }^{15}$ Volume and molecular rearrangements are constrained to occur within a thin slice of the simulation box, thereby greatly reducing the magnitude of the problem. Such moves have been shown to result in a fast equilibration of the density in NPT simulations of hard core and square-well chain systems, particularly for long chains at liquidlike densities. ${ }^{15,16}$ Note, however, that slab moves as originally proposed are only applicable to linear chains. We present in this work extensions of the method to enable simulation of branched and crosslinked polymers. These extensions are necessary because, whenever repositioned within the slab, branch linking sites require special moves to comply with the increased number of connectivity constraints.

In this study we present implementations of ECCB moves as applied to branched molecules with 3 and 4 branch linking sites. We then describe the extensions of slab volume moves to branched and crosslinked polymers. We apply these moves to simulate athermal and thermal (square-well) branched chains and we evaluate the effect of attractive interactions on the packing properties of such systems. We also present isothermal PV data for pure, ideal networks having a diamondlike topology of nodes and a discussion of our results. To the best of our knowledge, our results constitute the first systematic study of the PVT properties of polymer gels by computer simulation.

\section{THEORY}

In this study we consider flexible molecules formed by spherical sites connected by rigid bonds. Two types of nonbonded site-site interactions are considered, namely, hardcore (HC) and square-well (SW) potential energy functions

$$
\begin{aligned}
u_{\mathrm{HC}}(r) & =\infty, \quad r<\sigma \\
& =0, \quad r>\sigma, \\
u_{\mathrm{SW}}(r) & =\infty, \quad r<\sigma \\
& =-\epsilon, \quad \sigma \leqslant r \leqslant \lambda \sigma \\
& =0, \quad r>\lambda \sigma,
\end{aligned}
$$

where $r$ is the site-site distance, $\sigma$ is the site diameter, $\epsilon$ is the depth of the well, and $(\lambda-1)$ is the width of the well, which is set here to a value of 0.5 .

\section{A. ECCB moves for nodal sites}

Reference 14 presents a detailed description of the use of ECCB moves for branched molecules. Figure 1 shows a schematic molecular configuration around a 3-branch node. One of the most general prescriptions for performing mo-

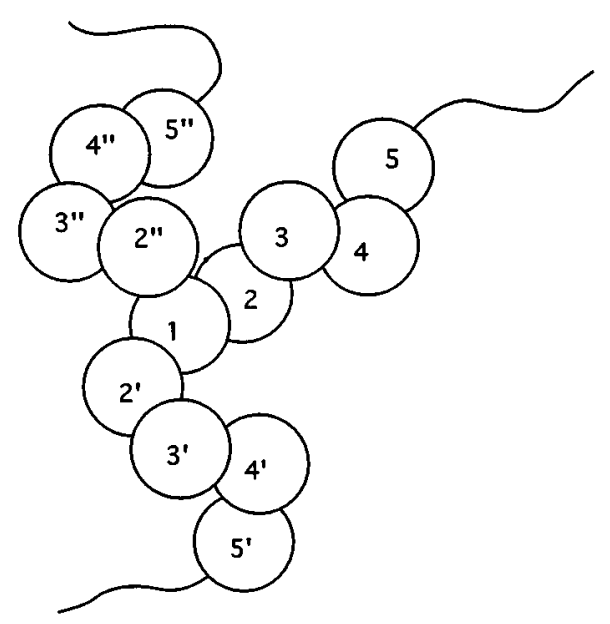

FIG. 1. Example of a local conformation of a chain portion around a trifunctional node (site 1). The numbering is arbitrary.

lecular rearrangements consists of proposing a simultaneous repositioning of an arbitrary section of the molecule, which may include nodes and multiple paths (or threads). For instance, in Fig. 1 the sites to be repositioned could include sites $3^{\prime}, 2^{\prime}, 1,2,2^{\prime \prime}, 3^{\prime \prime}$, and 4"; when these sites are temporarily deleted, the chain is left open at three points (sites $4^{\prime}$, 3 , and $5^{\prime \prime}$ ). In such a case the growing process first proceeds by closing two of the open sites (for instance, from $4^{\prime}$ to 3 ) and then continues by closing the third open site from the node (i.e., from 1 to $\left.5^{\prime \prime}\right) .{ }^{14}$ Such an approach can be equally implemented on $n$-branch nodes, where $n>2$. Note that in general the last site to be regrown cannot be an $n$ node if $n>4$.

The general approach just described is robust but somewhat involved in that it requires a systematic and reversible way of selecting different sections of the chain to be moved. Furthermore, a bookkeeping scheme would also have to be devised to handle the regrowing of multiple paths with all their possible variations. For this study we have instead implemented a variation of the general approach in which only a single path of sites is selected for repositioning. The single path may or may not contain a node; for instance, in Fig. 1 a path could consist of sites 2', 1, 2, and 3. A shortcoming of this particular variation is that nodes having at most 4 branches can be handled. Fortunately, such a limitation is not serious in practice because realistic molecules do not have coordination numbers $\left(N_{\text {co }}\right)$ above 4 . We now describe in detail the sequence of steps for such a method:

(1) Select at random the chain to be moved.

(2) Choose randomly a path of (connected) sites within the chain [the sites constitute a set $\left\{N_{s}\right\}$ ] and delete them temporarily. Let $N_{\text {cut }}$ be the number of sites to be repositioned and $N_{\text {open }}$ the number of sites left open when the $N_{\text {cut }}$ sites are deleted.

(3) If no nodal site belongs to $\left\{N_{s}\right\}$, two cases may occur. When an end-site $\in\left\{N_{s}\right\}$, an outer ECCB move (a standard CCB move) is performed and the growing sequence is such that the end-site is the last site to be repositioned. If no end-site $\in\left\{N_{s}\right\}$, then an inner ECCB move is performed. ${ }^{14}$ 
(4) The first site cannot be an end-site unless $N_{\text {cut }}=1$. The last site to be regrown cannot be a node with $N_{\text {co }} \geqslant 4$ (coordination number) because its position would be overspecified.

(5) For each site $k$ to be regrown, $m_{k}$ trial orientations of the bond vector are generated. Three cases can occur, depending on the number of sites to which site $k$ is currently connected:

(1) If connected to just one site, the orientations are chosen at random on a spherical surface.

(2) If connected to two sites, the orientations are chosen at random on a circumference (which represents the actual sampling space available to site $k$ ), thereby resulting in a simple "crank-shaft" move.

(3) If site $k$ has to be connected to three sites, then there are only two possible positions for site $k\left(m_{k}=2\right)$ which are fully defined by the geometric constraints that need to be satisfied. Site $k$ may sit at either side of the plane formed by the three existing neighbor sites. Such move is named TRIPODE and Appendix B gives formulas for the calculation of the new positions. By the rule given in step (4), no growing site will need to be attached to four sites.

(6) For each trial orientation of the growing site $k$, compute the distances between site $k$ and each of the $N_{\text {open }}$ sites which remain open at this point. The currently open sites form the set $\left\{N_{o}\right\}$ composed of $N_{d}$ sites. Note that $N_{d}<N_{\text {open }}$ always. A trial orientation is considered valid if the distances $d_{k, j}$ between site $k$ and each of the open sites $j$ satisfy the condition

$$
d_{\min } \leqslant d_{k, j} \leqslant d_{\max } \quad j \in\left\{N_{o}\right\},
$$

where $d_{\min }=\left(r_{k}+r_{j}\right)$ ( $r$ is the site radius) and $d_{\max }$ is the maximum physical separation that sites $k$ and $j$ can have and still permit closure of the chain section between them. Distance $d_{\max }$ is computed from geometric considerations; a detailed account of this issue is given elsewhere. ${ }^{14}$ For homonuclear chains with tangent or overlapping sites, analytical solutions for $d_{\max }$ are given in Appendix A.

(7) Provided that nonvalid orientations [according to criteria given by Eq. (2)] are assigned an infinite configurational energy, one of the trial orientations is chosen with probability

$$
W_{i}=\frac{w_{i}}{\sum_{k=1}^{m_{k}} w_{k}},
$$

where $w_{i}=\exp \left(-\beta U_{i}\right)$ is the Boltzmann factor for the $i$ th trial orientation in which growing site $k$ interacts with the rest of the system (except for the segments not regrown yet) with energy $U_{i}$, and $\beta=\left(k_{B} T\right)^{-1}\left(k_{B}\right.$ is Boltzmann's constant).

(8) If the regrown process fails because no valid or favorable configuration was found for any growing site, the move is rejected.

(9) If the repositioning of all the $N_{\text {cut }}$ sites is successful, the process is repeated (following the same sequence as before) to recover at each step the old configuration (i.e., the old orientations are included as one of the trial orientations, say the $i$ th, and chosen with probability $W_{i}$ ). Compute the corresponding weights using Eq. (4).

(10) The move is accepted with probability

$$
P_{\mathrm{ECCB}}=\min \left(1, \frac{J^{\text {new }}}{J^{\text {old }}} \frac{W_{\text {chain }}^{\text {old }} P^{\text {new }}}{W_{\text {chain }}^{\text {new }} P^{\text {old }}}\right),
$$

where $P$ is the probability of the state of the system, and

$$
W_{\text {chain }}=\prod_{i=1}^{N_{\text {cut }}} W_{i} \text {. }
$$

In Eq. (5) $J$ is the Jacobian determinant for the transformation of sampling space variables entailed by the growing process. The analytical evaluation of $J$ for different cases is given in Appendix C.

Note that the method outlined above corresponds to an implementation of the so-called mode-1 sampling for the trial orientation of a growing site $;{ }^{14}$ the alternative mode-2 sampling can be implemented following similar guidelines. Also note that in such a scheme the Jacobian correction is applied at the end of the process. A somewhat more effective method consists of incorporating this correction gradually, at each step of the growing process. This is done by modifying the $w_{i}^{\prime} s$ in Eq. (4) with the Jacobian determinant associated with each trial position (i.e., its effect one step ahead). In such a case, the Jacobian correction appearing in Eq. (5) drops out.

\section{B. Slab-volume moves for branched and crosslinked polymers}

Reference 15 describes two variations of sectional volume moves. Here we describe an extension of the simplest of them, the so-called slab method, to branched polymers and networks. The method consists of selecting a thin slice of the simulation box, i.e., a slab, and then proposing either a contraction or an expansion of the slab thickness. Since such a volume change can break bonds or cause overlaps between different sites of chain molecules, all sites within the slab are repositioned in such a way as to maintain connectivity constraints. The move is then accepted or rejected according to Metropolis-like criteria. The type of move experienced by sites within the slab depends on the type of configuration of the chain portions to which they belong. For instance, for linear chains, the chain could be completely (full) within the slab (i.e., the centers of all chain sites lie within the boundaries of the slab). More commonly, however, end portions (tail type), middle noncrossing portions (elbow type), or crossing portions (bridge type) are encountered in the slab. ${ }^{15}$ The repositioning of full, tail, and elbow portions does not present any difficulties because their internal configuration can remain unchanged and a simple concerted translation of sites maintains connectivity. For bridge sites, the internal configuration of the chain portion must be destroyed and rebuilt; to that end, we have developed a special move we call ISOPLANE (see description in Ref. 15).

For linear chains only single bridges can occur. For branched or crosslinked polymers, however, multiple-path 


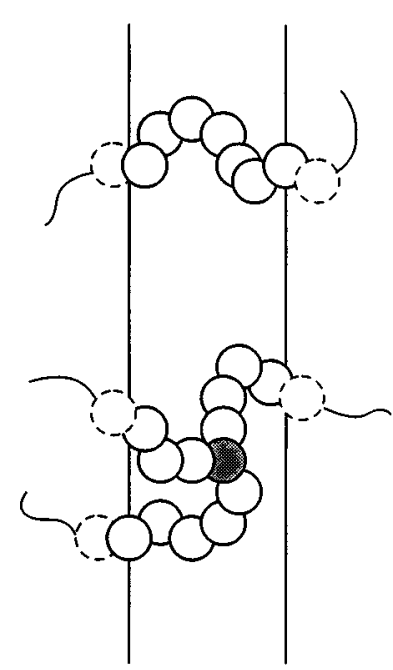

(a)

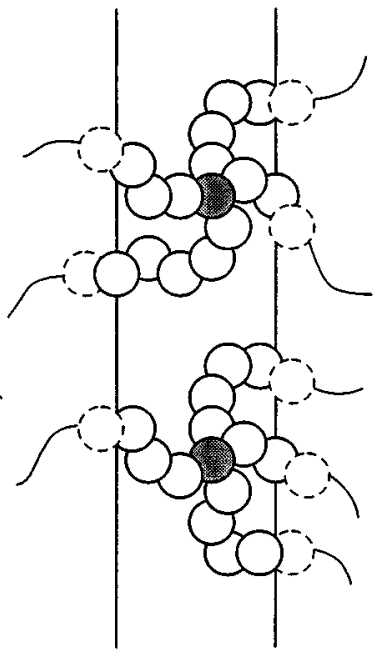

(b)
FIG. 2. Example of simple bridge conformations within the slab, (a) a single path bridge (linear chain portion) and a multiple path bridge originated by a trifunctional node; (b) multiple path bridges originated by a tetra-functional node in the chain portion. The vertical lines represent the slab walls.

bridges can occur, i.e., at some point in the bridge several parallel paths can be present. A few simple multiple-path bridges are illustrated in Fig. 2. The repositioning of bridge sites involves three steps:

-Disconnect or decouple the bridge by deleting temporarily some of its sites, which we refer to as dangling sites.

- Reposition the remaining sections of the bridge by treating them as full, elbow or tail portions.

- Reposition the dangling sites to restore the architecture of the chain portion.

If a dangling site has $N_{\mathrm{co}}=2$, then it can be repositioned with a usual ISOPLANE move ${ }^{15}$ (provided that none of the two neighbor sites is also a dangling site). In principle, any complex multiple-path bridge can be disconnected by only choosing bifunctional dangling sites as long as the following rules are satisfied:

(1) There are no neighbor nodes.

(2) The slab is made thick enough so that at least two sites are present for any chain portion.

These rules ensure that any chain portion will have at least one bifunctional site. Although choosing always bifunctional dangling sites is a conceptually simple approach, it proved to be difficult to implement. To understand the reason for this let us consider the bridge illustrated in Fig. 3(a). Possible choices for dangling sites might be the sets: $\{1,11\},\{3,11\}$, $\{1,9,10\}$, etc. In principle, a set with the least number of dangling sites should be preferred. A successful identification of possible sets depends on a proper classification of all sites in the bridge and a systematic topological analysis of all possible combinations of candidate sites. While such a scheme is viable, it would be quite cumbersome for the com-

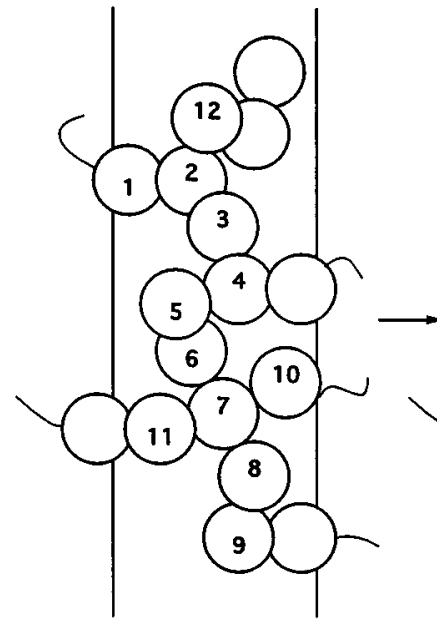

(a)

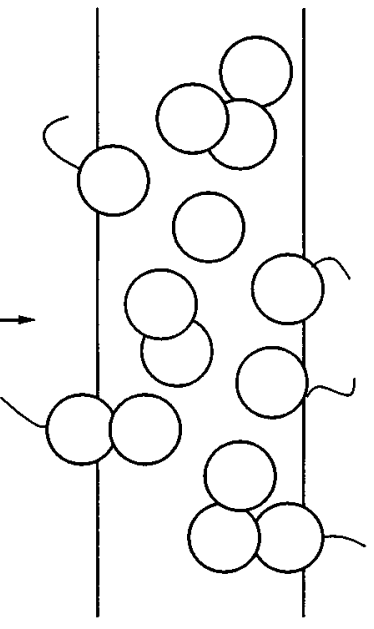

(b)
FIG. 3. Example of a multiple path bridge when more than one node is present in the chain portion within the slab. (a) Original conformation of the bridge, sites 2, 4, and 7 are the nodes. (b) Conformation that results when the nodes have been deleted; the bridge has been disconnected, i.e., there is no path joining sites at both sides of the slab walls.

plex bridges (with numerous, tortuous paths) that arise in highly branched chains and crosslinked polymers (the number of possible sets grows rapidly).

A simpler approach for selection of dangling sites consists of always choosing the nodes. To apply this method the two rules aforementioned must also be satisfied. Of course, if no nodes are present in the bridge, we choose any of the bifunctional sites at random and perform a conventional ISOPLANE move (after the neighbor sites have been moved). If a dangling site is a tri-functional node, it is repositioned with a TRIPODE move. If a dangling site is a tetrafunctional node, then one of the bifunctional neighbor sites must also be regarded as a dangling site (from the two rules given before, there has to be at least one such site); the node is repositioned first by using a TRIPODE move (since it temporarily behaves as a tri-functional node) and then the bifunctional neighbor site is repositioned through an ISOPLANE move. Although nodes with higher functionality than four could be handled by a similar approach, they are not considered in this study. Figures 3(a) and 3(b) illustrate how the application of this method disconnects the bridge by breaking it into several pieces. Note that, by deleting all nodes $\{2,4,7\}$, we create redundant dangling sites; for instance, it would suffice to delete two nodes, either $\{2,7\}$ or $\{4,7\}$. However, the additional expense of having more dangling sites is compensated by avoiding the topological analysis that would be required to optimize the selection process. It can be shown that the simple prescription of using nodal dangling sites will always work, no matter how complex the bridge. Note that, to apply this method, a third rule must be satisfied (in addition to the two rules aforementioned): There have to be at least two (bifunctional) sites between two tetrafunctional nodes. 
The steps required by this method are summarized as follows:

(1) Choose one axis at random. For simplicity, relabel the chosen axis as $x$.

(2) Locate the slab randomly along the $x$ axis and choose its thickness $h_{\text {old }}$. The value of $h_{\text {old }}$ must be large enough so that it guarantees that chain portions of at least two (neighbor) sites will be present.

(3) Identify and delete all sites whose centers lie within the slab and identify all single (one-path) bridges. For these single bridges choose at random one site to be left dangling. Also tag all nodes within the slab as dangling sites. For tetra-functional nodes, also tag one of its bifunctional neighbor sites (within the slab) as a dangling site.

(4) Propose a volume change by choosing a new slab thickness, i.e., $h_{\text {new }}=h_{\text {old }}+\delta h$, where $\delta h$ is chosen randomly in a preselected range $\left[-\delta h_{\max },+\delta h_{\max }\right]$.

(5) With the exception of the dangling sites, reposition all sites within the slab. This is done by maintaining their $x$ distance to one of the walls constant while preserving chain connectivity.

(6) Reposition all nodal dangling sites using TRIPODE moves. Since a TRIPODE move generates two possible positions, to satisfy detailed balance we always choose the position that lies on the same side of the plane formed by the three (already repositioned) neighbors as in the old configuration.

(7) Reposition the bifunctional dangling sites with ISOPLANE moves.

(8) If during repositioning the center of a site falls outside the slab, the whole move is rejected. Otherwise, the move is accepted with probability.

$$
\begin{aligned}
P_{\text {accept }}= & \min \left\{1,\left[\prod_{i=1}^{N_{\mathrm{ds}}} \frac{\mathbf{J}_{i}^{\text {new }}}{\mathbf{J}_{i}^{\text {old }}}\right]\right. \\
& \left.\times \exp \left[-\beta \Delta U-\beta P\left(V_{\text {new }}-V_{\text {old }}\right)\right]\right\},
\end{aligned}
$$

where $\Delta U$ is the change in energy produced by the volume change over the system and $\mathbf{J}_{i}^{\text {new }} / \mathbf{J}_{i}^{\text {old }}$ is the ratio of Jacobian determinants associated with the repositioning of the $i$ th dangling site (the total number of dangling sites in the slab is $N_{\text {ds }}$ ).

\section{SIMULATIONS}

The methods described in the previous section and our newly developed algorithms were tested by comparing some of our new simulation data with those obtained by standard simulation methods (whenever available). Thorough tests of ECCB-type moves and slab moves for linear chains have been reported earlier. ${ }^{14,15}$ Select NPT simulation points for athermal and SW chains and for tri and tetra functional branched chains were performed both by slab moves and standard volume moves. In all cases we found complete agreement between the results of both methods; any ob-

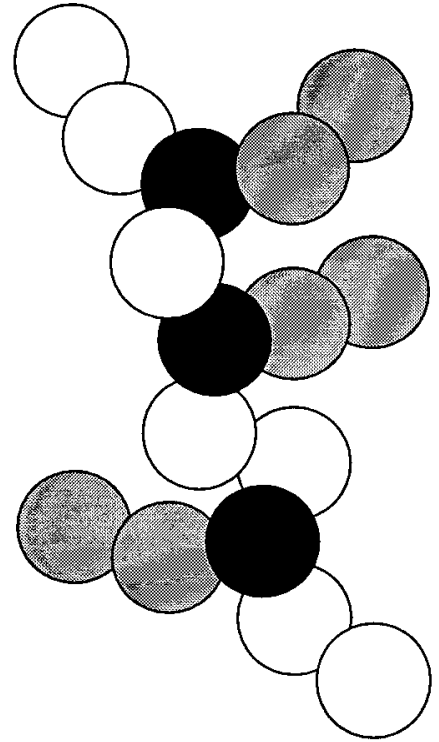

FIG. 4. Topology of the branched 16-mer chain used in our simulation. The black beads are the nodes and the shaded beads belong to the branches.

served differences were well within the error bars of the simulations. It is noted that the omission of the Jacobian determinants in the acceptance criteria for the volume moves can produce surprisingly large errors; in some cases as high as $100 \%$ of the true equilibrium value of the density.

\section{A. Athermal and thermal branched molecules}

We studied a fully flexible branched molecule of 16 tangential beads. As shown in Fig. 4, this chain has three small branches of two sites each, which are not symmetrically located along the backbone. This chain topology provides a relatively high concentration of nodes. A number of simulations were carried out to compare the behavior of our branched chain model and simple linear flexible chains of 16 segments; we conducted isothermal-isobaric simulations of both athermal and SW chains. The total number of chains in these simulations ranged from 40 (for the lower densities) to 80 (for the higher densities). Molecular rearrangements were performed using ECCB moves; for branched chains we employed the single-path method described in the previous section. We also used whole molecule translations, particularly at low densities. Volume fluctuations were performed using slab moves according to the guidelines given in the previous section.

We also conducted phase equilibrium simulations for linear and branched SW 16-mers. We adopted a variation of the NPT- $\mu$ method of Moller and Fischer. ${ }^{19}$ This method is based on a series of NPT simulations and chemical potential calculations; details regarding its implementation can be found elsewhere in Ref. 16.

\section{B. Ideal networks}

A realistic representation of a crosslinked polymer would necessarily involve a highly irregular network. Such a network typically has dangling ends, a "patchy" distribution 
of nodes, self-loops, etc. ${ }^{20}$ For this purpose, relatively large systems should be generated in some physical fashion; the resulting system should exhibit a realistic distribution of all topological entities and provide a truthful representation of the macrosystem. This is the approach followed by Kremer et al. ${ }^{9,10}$ who prepared polymer networks by randomly crosslinking a melt of linear chains with polyfunctional ends. Topological irregularities, however, have important effects on the thermodynamic properties of networks; it would therefore be highly desirable to isolate them from other, more fundamental characteristics of such systems. Everaers and $\mathrm{Kremer}^{20,21}$ have very recently studied rubber elasticity on "chemically" defect-free polymer networks. For this purpose, they interpenetrate a number of diamondlike networks. As its name suggests, a diamondlike network is one in which the connectivity of the strands corresponds to that of a diamond lattice. The interpenetration of several such networks is an artifact introduced to achieve a meltlike density and a realistic concentration of crosslinks (nodes).

In this study we investigate single diamond networks, i.e., we do not resort to interpenetrating structures. We achieve the desired concentrations of nodes by simply compressing the system. We believe that a single diamond network provides a more adequate reference system for fundamental studies; the interaction and positioning of the different networks in the Everaers and Kremer's model introduces additional topological effects that we prefer to avoid in our work.

We used simulation boxes having 8 diamond unit cells $(2 \times 2 \times 2)$, that is, there were 64 nodes or crosslinks $\left(N_{\text {nodes }}\right)$ in the box. Preliminary studies with smaller and larger systems revealed that finite size effects were negligible for such a system size. Note that much smaller systems can be used by working with ideal networks (as opposed to random networks) because, in a sense, even a unit (periodic) cell is representative of the system. The total number of sites in the simulation box $\left(N_{t}\right)$ can be computed from

$$
N_{t}=N_{\text {nodes }}\left(1+2 N_{\text {str }}\right) \text {, }
$$

where $N_{\text {str }}$ is the number of sites in a single strand between two nodes. In the networks studied here $N_{\text {str }}$ is constant (homogeneous strand length).

Initial configurations were generated as follows. Given some specified value of $N_{\text {str }}$ and a packing fraction $\eta$, we first computed the box size and then positioned the nodes in a regular diamond lattice arrangement. The strands were subsequently grown using ECCB-like moves. Such an approach was found to be practical for densities of up to $\eta=0.25$. Systems at higher densities were prepared by compressing the system through NPT simulations. The parameters of the simulation were adjusted in such a way as to preserve (roughly) the cubic symmetry of the simulation box even after many volume fluctuations.

\section{RESULTS AND DISCUSSION}

Figure 5 shows our simulation results for linear and branched hard-core chains. Simulation results for linear

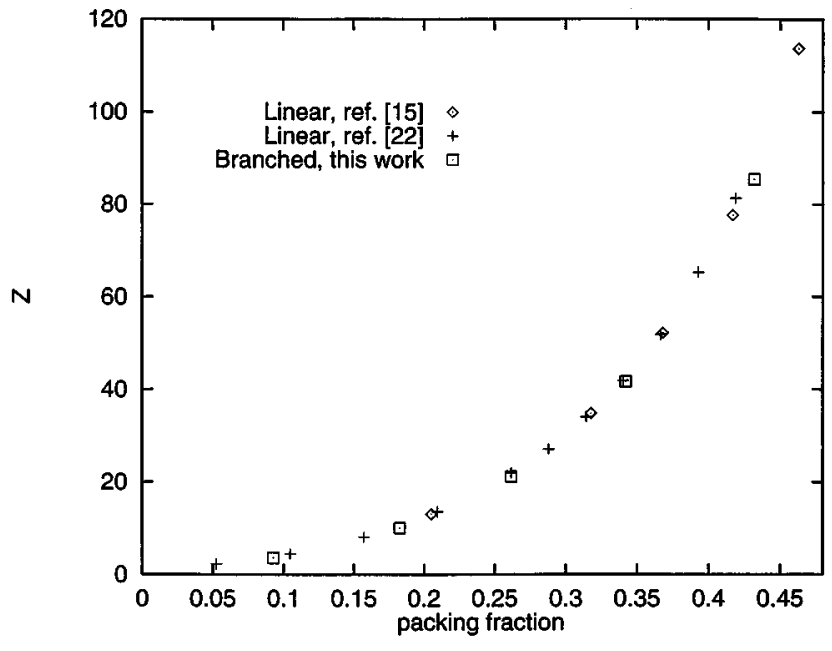

FIG. 5. Compressibility factor for the linear and branched hard-sphere chains of 16 segments. Our simulation data for the linear chain are from Ref. 15 .

chains from Ref. 22 are also included in that figure. It is observed that, within the uncertainty of the data, the curves of compressibility factors are indistinguishable. This relative lack of sensitivity of the PV properties of hard-core molecules to the details of chain architecture is consistent with previous findings for linear and starlike molecules. ${ }^{7,17,18}$

In Table I and Fig. 6 we present our results for branched and linear SW chains. For comparison, we also give simulation data for linear 16-mer SW chains from Ref. 23 and the predictions of an equation of state that we proposed recently. ${ }^{16}$ The reduced temperature in both cases was fixed at $T^{*}=k_{B} T / \epsilon=3$, which corresponds to supercritical conditions $\left(T_{c}^{*} \approx 2.3\right.$, see below). Our simulation data for linear 16-mers and those from Ref. 23 exhibit small but systematic deviations. These differences are important when comparing data for the linear chains to those for the branched chains, which also fall in close vicinity. We believe that our results are more reliable because much longer calculations have been used to generate our data. Also, the predictions of our equation of state from Ref. 16 (which is expected to be highly accurate for linear 16-mers) are in good agreement with our simulation data. Our results for branched SW chains are indistinguishable from those for linear chains (within error bars).

TABLE I. Results of NPT simulations for linear and branched SW chains of 16 segments.

\begin{tabular}{lcc}
\hline \hline & \multicolumn{2}{c}{$\eta$} \\
\cline { 2 - 3 }$P^{*} / T^{*}$ & Linear & Branched \\
\hline 0.0125 & $0.092(7)$ & $0.088(6)$ \\
0.047 & $0.179(7)$ & $0.181(7)$ \\
0.175 & $0.263(5)$ & $0.262(4)$ \\
0.388 & $0.309(5)$ & \\
0.67 & $0.341(4)$ & $0.341(4)$ \\
2.70 & $0.428(2)$ & $0.429(2)$ \\
\hline \hline
\end{tabular}




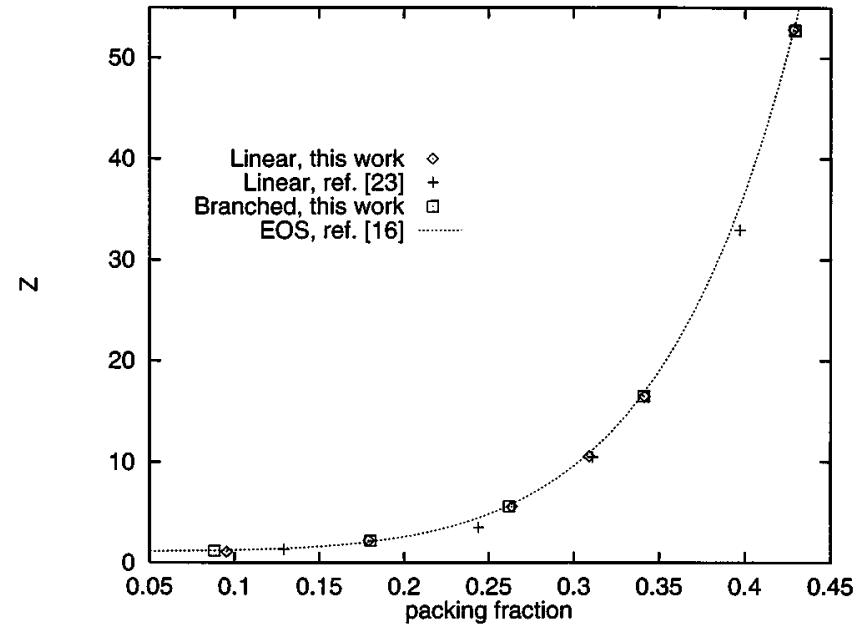

FIG. 6. Compressibility factor for the linear and branched SW chains of 16 segments at $T^{*}=3$. The line represents the predictions of an equation of state based on equations for two reference fluids (4-mer and 8-mer) as reported in Ref. 16.

While PVT data do not reveal noticeable differences (at least at the supercritical isotherm studied here), phase equilibrium simulations for these chains are more informative. The results are shown in Table II and Fig. 7. The critical properties were estimated from the rectilinear diameter rule and the scaling law for the coexistence curve. ${ }^{24}$ It is interesting to note that the coexistence curves for both isomers exhibit systematic differences. While at temperatures well below the critical the coexistence data for both systems seem to overlap, at higher temperatures the vapor phase density is larger and the liquid density is lower for branched chains. Figure 8 shows the vapor pressure curves for both isomers, which confirm the higher volatility of branched chains. These observations are in agreement with experimental observations for non-polar polymers and simple alkanes; (liquid) branched isomers have lower densities ${ }^{25}$ and lower boiling points than linear chains. The lower density of branched chains is explained by a steric effect which hinders effective packing of the molecules. Since branch-linking sites and

TABLE II. Results of phase equilibrium simulations for SW chains. Critical values are estimates (see the text).

\begin{tabular}{ccccc}
\hline \hline$T^{*}$ & $\eta_{\text {liq }}$ & $\eta_{\text {vap }}$ & $\beta \mu_{\text {liq }}^{F}$ & $\beta \mu_{\text {vap }}^{E}$ \\
\hline $16-$ mer & Linear & & & \\
1.95 & $0.310(5)$ & 0.0015 & -5.6 & -0.14 \\
2.00 & $0.295(4)$ & 0.002 & -5.2 & -0.12 \\
2.05 & $0.280(5)$ & 0.0028 & -4.8 & -0.18 \\
2.10 & $0.268(6)$ & 0.006 & -4.1 & -0.34 \\
2.15 & $0.250(8)$ & 0.013 & -3.5 & -0.48 \\
2.20 & $0.220(9)$ & 0.018 & -3.1 & -0.6 \\
2.33 & \multicolumn{2}{c}{ Critical $^{*}$} \\
$16-$ mer & Branched & & \multicolumn{2}{c}{} \\
2.00 & $0.290(4)$ & 0.005 & -4.3 & -0.22 \\
2.05 & $0.274(5)$ & 0.007 & -4.0 & -0.30 \\
2.10 & $0.250(7)$ & 0.009 & -3.7 & -0.38 \\
2.15 & $0.228(8)$ & 0.016 & -3.2 & -0.51 \\
2.25 & \multicolumn{2}{c}{0.104} & \multicolumn{2}{c}{ Critical $^{*}$} \\
\hline \hline
\end{tabular}

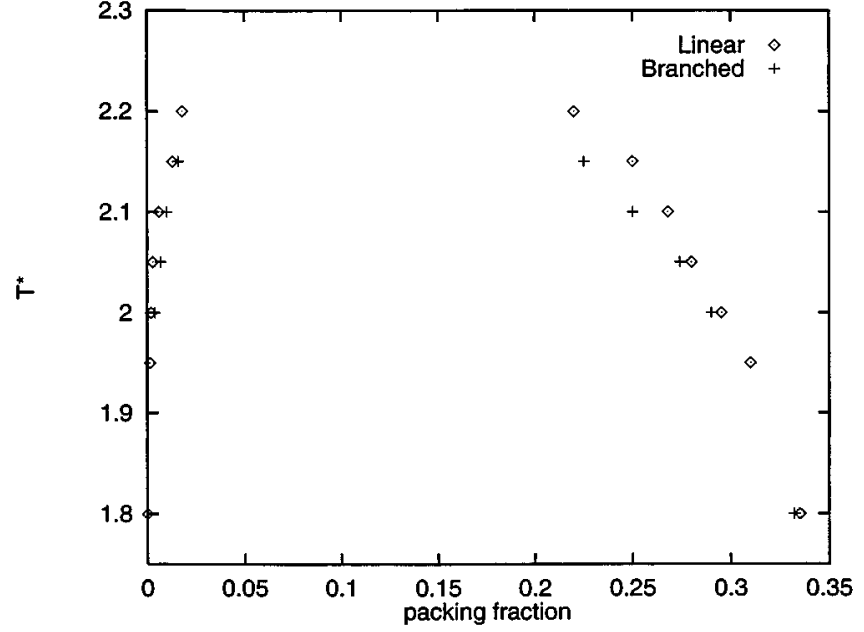

FIG. 7. Coexistence data for linear and branched SW chains of 16 segments.

their neighbors on a given chain have a lower probability of interacting with sites from other chains, the magnitude of intermolecular interactions is somewhat reduced, thereby promoting a higher volatility of the molecules. Since the overall shape of a branched molecule tends to be more spherical, its effective surface area is reduced and intermolecular attractions are weakened. ${ }^{26}$ It is more difficult to explain why these differences are not observed at supercritical conditions or at temperatures well below the critical. It would appear that, for the molecular models studied in this work, any physical differences are rather small and only near critical conditions are such differences amplified. In real molecules the differences in PVT properties between branched and linear molecules are typically larger; presumably our fully flexible model (with freely rotating branches) does not properly represent some of the configurational constraints introduced by real branching.

Figure 9 shows a snapshot of the initial configuration of a perfect network with a strand length of 10 segments at

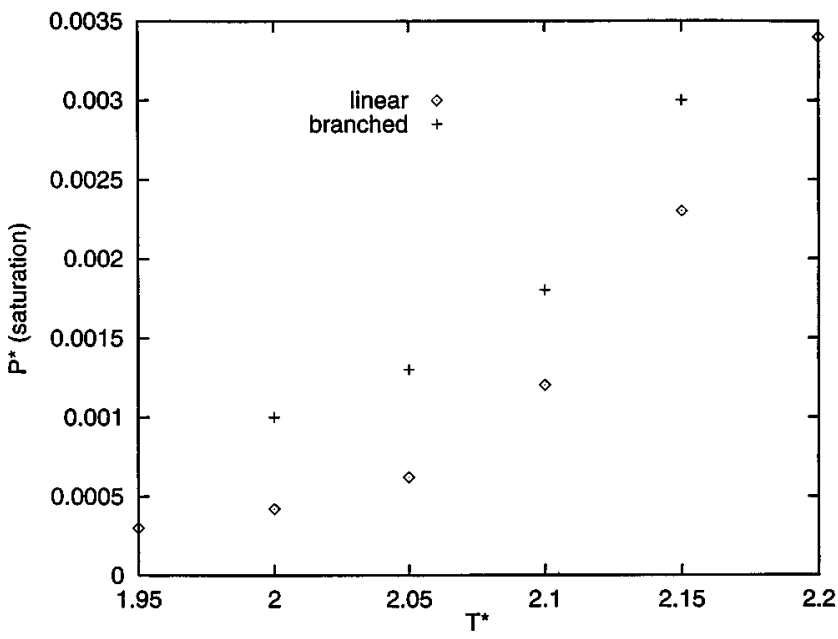

FIG. 8. Saturation pressure (in reduced units) for linear and branched SW chains of 16 total segments. Values estimated from phase equilibrium simulations. 


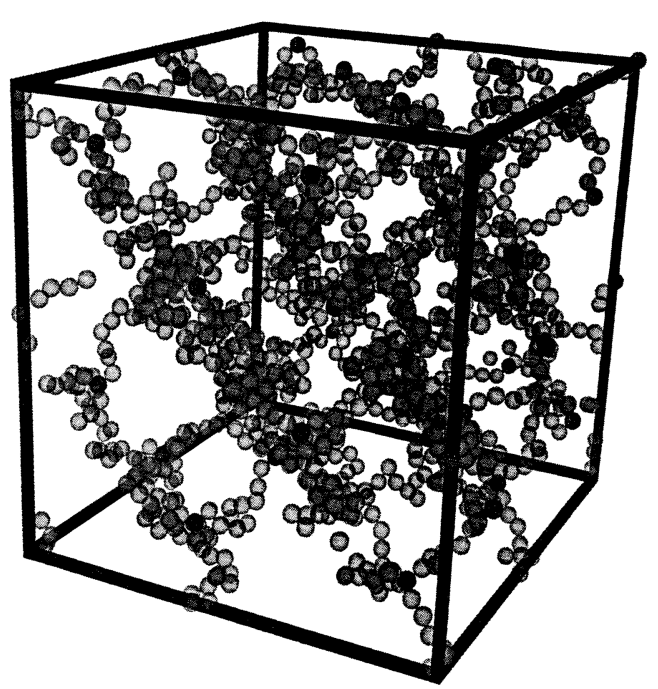

FIG. 9. Snapshot of the initial configuration of a perfect network with 10 segments per strand at $\eta=0.07$. The darker beads are the tetra-functional nodes which are (initially) placed in the box with a diamond lattice symmetry. There is a total of 64 nodes in the simulation box.

$\eta=0.07$. Table III and Fig. 10 give our simulation results for perfect athermal networks. The compressibility factor per site $(\bar{Z})$ is defined as $(\pi / 6) P^{*} / T^{*} \eta$, where $\eta$ is the packing fraction, $P^{*}=\sigma^{3} P / \epsilon$ is the reduced pressure, and $T^{*}=k_{B} T / \epsilon$ is the reduced temperature (the factor $P^{*} / T^{*}$ is preset in the NPT simulations). We present results for strand lengths of 3 and 10 segments. For comparison, simulation results for a star molecule of 21 total segments are also shown. ${ }^{7}$ This star molecule has 4 arms of 5 segments each; it has been chosen to represent a broken perfect network of 10 segments per strand. It is observed that, for intermediate to high densities, the $\bar{Z}$ curves for the networks follow the same trends as those of the broken polymer (which is in fact analogous to the trends for linear chains). A distinctive feature of the volumetric behavior of networks appears at low to intermediate densities, where the curve crosses the $P^{*}=0$ line and dives down to negative pressures. In contrast to multichain systems, whose pressure goes to zero as the density goes to zero, in a network the pressure must necessarily go to very large negative values when the density is decreased. The reason for this is that, for our system, a negative pressure is consistent with exerting an external force to expand

TABLE III. Results of NPT simulations for athermal perfect networks.

\begin{tabular}{ccccc}
\hline \hline \multicolumn{2}{c}{$N_{\text {str }}=10$} & & \multicolumn{2}{c}{$N_{\text {str }}=3$} \\
\cline { 1 - 2 } \cline { 5 - 5 } \cline { 5 - 5 }$P^{*} / T^{*}$ & $\eta$ & & $P^{*} / T^{*}$ & $\eta$ \\
\hline-0.02 & $0.013(2)$ & & -0.25 & $0.051(5)$ \\
0.0 & $0.064(2)$ & & -0.07 & $0.099(3)$ \\
0.05 & $0.127(3)$ & & 0.0 & $0.139(9)$ \\
0.30 & $0.220(3)$ & & 0.05 & $0.169(8)$ \\
1.0 & $0.306(2)$ & & 0.30 & $0.237(6)$ \\
2.0 & $0.365(6)$ & & 0.80 & $0.299(6)$ \\
3.5 & $0.414(3)$ & & 2.0 & $0.374(5)$ \\
& & & 3.5 & $0.422(4)$ \\
\hline \hline
\end{tabular}

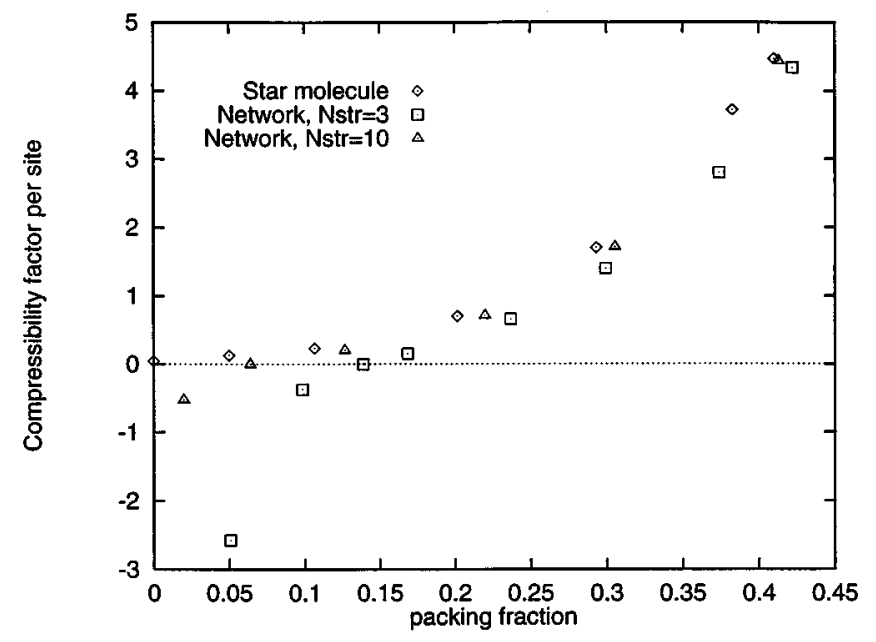

FIG. 10. Results of NPT simulations for hard-core perfect networks with 3 and 10 segments per strand. Results for a 4-arm star molecule with 5 segments per arm are also plotted (Ref. 7). The compressibility factor per site is defined as $P^{*} / T^{*} \rho_{s}$, where $\rho_{s}$ is the site density.

the system to a certain density. To gain some insight into how an isotropic, negative pressure can be exerted on the network, it is instructive to make the analogy to the osmotic pressure that arises in a solvent-gel system; such pressure is in fact negative and tends to expand (swell) the network. To the best of our knowledge, this work constitutes the first simulation of the compressibility of a pure network. It is therefore difficult to know whether or not negative pressures have been observed in the past.

For our perfect networks, there is a limiting low density, $\eta_{c}$, for which the strands are fully extended and no further expansion is possible without breaking bonds; if no strands are entangled, the fully extended network adopts a diamondlike structure with,

$$
\eta_{c}=\frac{\pi \sqrt{3}}{16} \frac{\left(1+2 N_{\mathrm{str}}\right)}{\left(1+N_{\mathrm{str}}\right)^{3}}
$$

i.e., only in the limit of large $N_{\text {str }}$ does $\eta_{c} \rightarrow 0$.

It is interesting to analyze the conditions for which $P^{*}=0$. In the absence of an external pressure, a system of uncrosslinked athermal molecules maximizes its entropy by expanding infinitely, i.e., by separating the molecules as much as possible (intermolecular interactions restrict the molecular motion). In a network, however, a separation of strands favors the entropy of the system only up to certain point. In fact, as individual strands are stretched their mobility becomes restricted (the actual free volume available to them is reduced); at the limit of maximal extension, only one configuration is available to a fully aligned strand and its entropy reaches a minimum. The density at which $P^{*}=0$, $\eta_{0}$, is representative of the equilibrium between these two competing entropic effects: the beneficial effect of unpacking the network and the detrimental effect of stretching the strands. Figure 11 shows our simulation data for $\eta_{0}$ and the curve for $\eta_{c}$, as computed from Eq. (9), as a function of the inverse strand length. As expected, $\eta_{0}>\eta_{c}$ and both curves seem to converge towards $\eta=0$ when $N_{\text {str }} \rightarrow \infty$. The entropic effect of strand extension begins to dominate sooner (i.e., at 


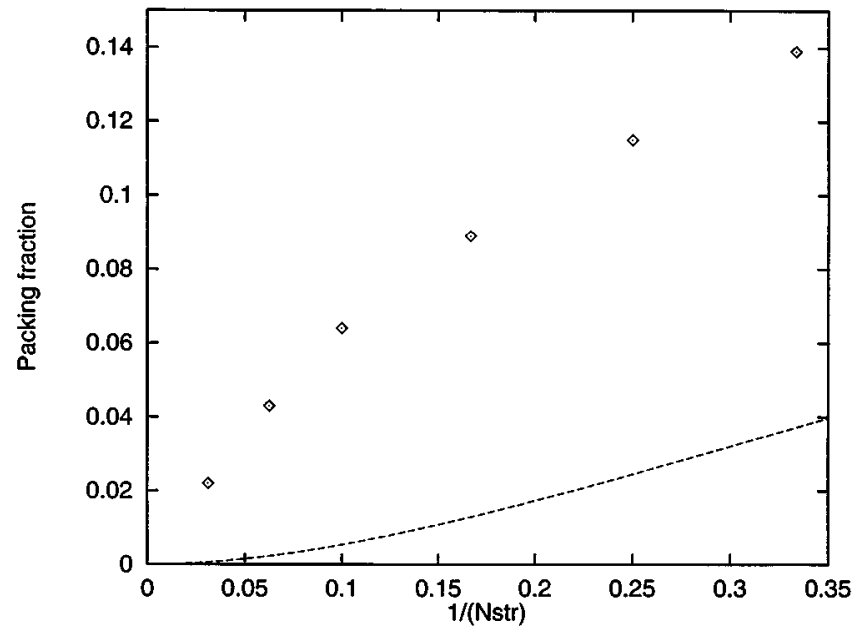

FIG. 11. Plot of simulation results of packing fraction at zero pressure, $\eta_{0}$, and the calculated minimum achievable packing fraction, $\eta_{c}$ [Eq. (9)] as a function of the inverse strand length, $N_{\text {str }}^{-1}$. The simulation points for $\eta_{0}$ correspond to strand lengths of $3,4,6,10,16$, and 32 segments.

higher densities) for networks of shorter strands. This is because a perfect network with shorter branches has a reduced mobility and less entropy; in the limit of $N_{\text {str }}=0$, the network behaves as a rigid matrix.

The different behavior of the network and its broken counterpart (i.e., the 4-arm star 21-mer) can be further elucidated by comparing their internal structure. Figures 12 show several site-site radial distribution functions for the 10 -site strand network at $\eta=0.064$ (which corresponds to $P^{*}=0$ ). To avoid large spikes in the distribution functions, bonded neighbor sites have not been included in their calculation. The distribution functions shown are $g 11, g 12$, and $g 22$ which correspond to the node-node, node-strand, and strand-strand pairs, respectively. Figures 12 include two types of strand-strand distribution functions: one for sites on different strands $\left(\mathrm{g} 22^{\prime \prime}\right)$ and another for sites on the same strand $\left(g 22^{\prime}\right)$. The analogous distribution functions $g 11$ and $g 12$ for the star molecule at the same density are almost indistinguishable from those of the network (for clarity, results for the star are not shown). However, since the strand length in the network is not the same as the arm length in a star molecule, a direct comparison of their $g 22$ distribution functions would not be too informative. Some insight into the relative differences between these two materials can be obtained if, to emulate the star molecule structure, we nominally separate the network strands into two identical arms of 5 sites each; the distribution functions generated (in the network) with this convention are labeled $g^{*} 22$. Figure 12(b) shows a comparison between the $g * 22$ 's of the network and the $g 22$ 's of the star system. In the network, there is a lower concentration of sites from the same arm around a given strand-site $\left(g^{*} 22^{\prime}\right)$ than in the star system. This result reflects the fact that, whenever sites of the same first portion of a network strand approach each other, the second portion of the same strand (nominally different arms) tends to be stretched. This coupling (nonexistent in the star system) has a detrimental effect on the average local concentration of

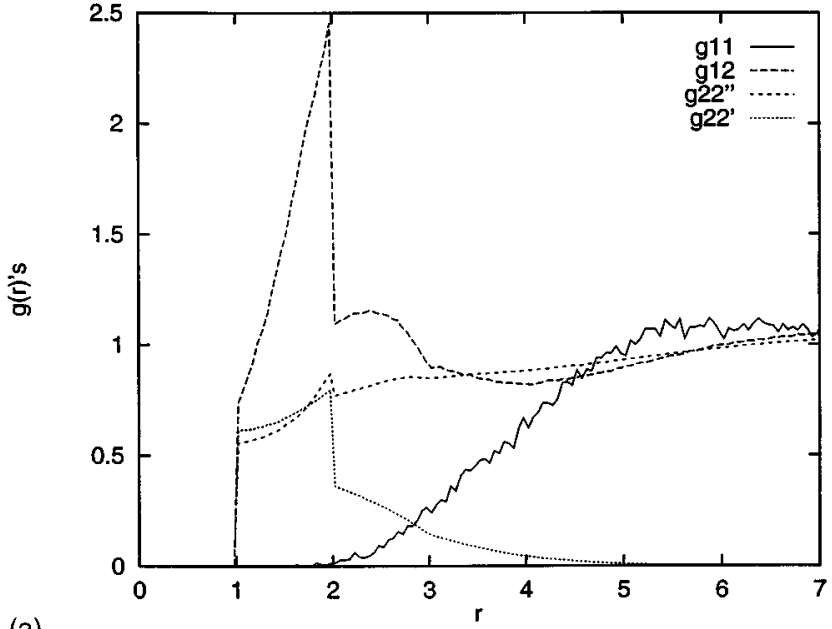

(a)

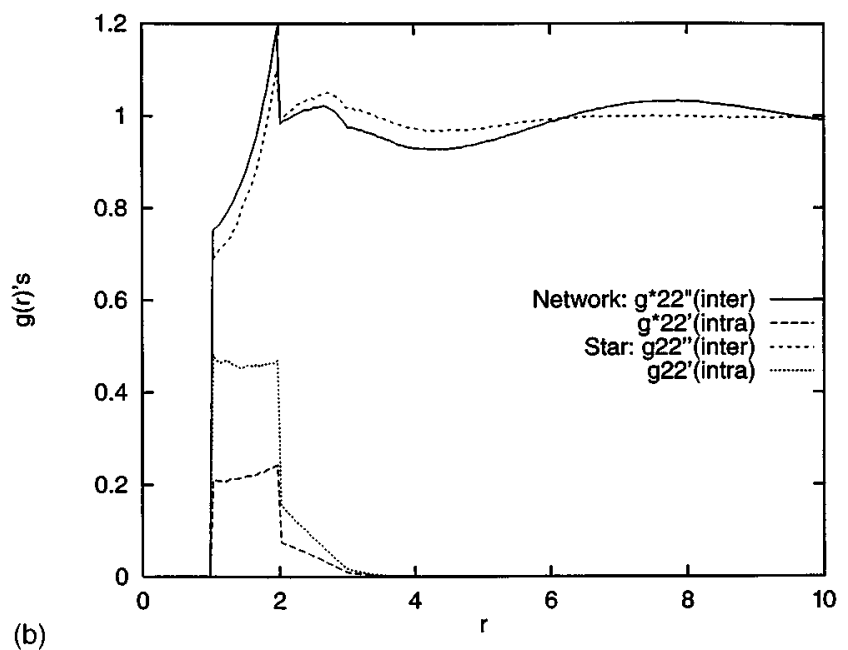

FIG. 12. Site-site radial distribution functions at $\eta=0.064$ in (a) a hard-core perfect network of 10 sites per strand and (b) the same hard-core network and a 4-arm star of 21 sites. The distribution functions are labeled as $g \alpha \beta$ where $\alpha$ and $\beta$ identify the type of interacting site: $1=$ tetrafunctional node, $2=$ strand or arm site. The distribution functions $g * 22$ correspond to armarm site pairs in a network where the sites of each individual strand are assigned to two arms of equal length. In all cases, bonded neighbor sites are not included in evaluating the histograms.

intra-arm sites. Note, however, that this small intra-arm population in the network is deceiving; as a closer inspection of Figs. 12 reveals, a significant portion of the inter-arm population $\left[g^{*} 22^{\prime \prime}\right.$ in Fig. 12 (b) $]$ is actually a result of (true) intrastrand contributions $\left[g 22^{\prime}\right.$ in Fig. 12(a)]. Although $g^{*} 22^{\prime \prime}$ (network) and $g 22^{\prime \prime}$ for the star system are much more alike than $g^{*} 22^{\prime}$ and $g 22^{\prime}$, the distribution function for the network has a longer range.

The additional structure observed in $g * 22^{\prime \prime}$ practically disappears at higher densities (i.e., $\eta>0.15$ ); such a feature seems to indicate the onset and predominance of entropic effects associated with strand stretching in the network. The oscillations of $g^{*} 22^{\prime \prime}$ reflect the long range effects (commensurate with the strand length) of the interaction between sites from the two arms of a same strand. Such effects are negligible at high densities, when the strands tend to be folded. However, they become stronger as the density is decreased 


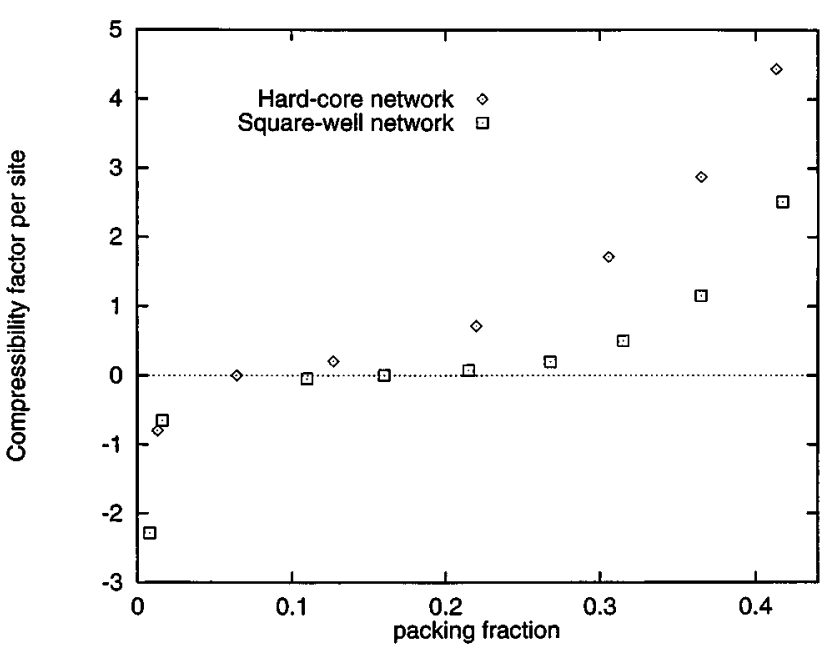

FIG. 13. Results of NPT simulations for the hard-core and SW perfect network with 10 segments per strand. The temperature for the SW network simulations is $T^{*}=2.5$. The compressibility factor per site is defined as $P^{*} / T^{*} \rho_{s}$, where $\rho_{s}$ is the site density.

and the strands are stretched. For brevity, other secondary long range features of the interarm and interstrand distribution functions, which are caused by the interaction of sites on strands connected to the same node, are not discussed any further in this work.

Attractive site-site interactions could lead to a number of interesting phenomena, particularly at conditions where phase transitions may occur. In this initial study, however, we restricted our efforts to illustrating the effects of attractive forces on $\eta_{0}$. For this purpose, we simulated a perfect network of 10-segment strands formed by SW sites. The temperature was $T^{*}=3$, which is high enough to prevent a phase transition from occurring. The results are given in Fig. 13. The value of $\eta_{0}$ was increased significantly, from $\eta_{0}=0.064$ for the hard-core network to $\eta_{0}=0.16$ in the SW network. This increase reflects the fact that the extension of the strands is now even more unfavorable, because attractive forces tend to keep sites together. The entropic effects of strand stretching associated with low densities are reinforced by energetic effects, leading to denser equilibrium configurations at $P^{*}=0$. A nearly flat region, which spans the range $[0.05<\eta$ $<0.20]$, is observed in the $\bar{Z}-\eta$ isotherm around $\eta_{0}$; this behavior suggests the proximity of a critical point, where the first two derivatives of $P^{*}$ with respect to density would vanish.

\section{CONCLUSIONS AND FUTURE WORK}

A variation of the extended continuum configurational bias method was developed in detail to efficiently generate molecular rearrangements in branched and crosslinked polymers. Linking sites with a coordination number of up to four can be simulated using this method. A simple move, denoted TRIPODE, was fully implemented to reposition sites which need to be reattached to three pre-existing neighbor sites. Isothermal-isobaric simulations were implemented by relying on the molecular moves aforementioned and on slab moves, for which volume changes and molecular rearrangements are performed simultaneously within a thin slice of the simulation box. The multiple coupling of different molecular portions in a network precludes that independent translations of those portions be performed along with a volume change. Since the topological conformations of bridging chain portions can become very complex, we have implemented a general method that temporarily decouples all bridge-type conformations within the slab by deleting selected sites. All bridges are subsequently restored by using simple, deterministic moves.

We performed a number of NPT simulations to compare the PVT behavior of both athermal and thermal models of linear and branched chains. Our results support the observation that branching has a negligible effect on the single-phase thermodynamic properties of fully flexible molecular models. Phase equilibrium simulations, however, exhibit more clearly the effects of branching, particularly close to critical conditions.

Our simulations of perfect (diamondlike) networks revealed an interesting competition between two entropic effects associated with the expansion of the network. On the one hand, a positive effect results from the increased availability of free volume (uncrowding of space) encountered at lower densities. On the other hand, a negative entropic effect is introduced as the strands become more and more stretched.

The simulation methods and results presented here provide a starting point for a more systematic and thorough study of the physics of systems containing branched and crosslinked polymers. Variations in the polymer topology and potential energy models will in all likelihood bring about a number of interesting physical phenomena. For instance, our preliminary simulations have revealed the existence of phase transitions in pure networks. We are currently investigating gel swelling by simulating the phase equilibrium of a network-solvent system in different ensembles.

A semiquantitative description of the PVT behavior in networks can be readily implemented by resorting to meanfield theories for rubber elasticity and gel swelling such as those of Wall and Flory. ${ }^{27}$ A detailed theoretical analysis of such a description will be presented in a future publication.

\section{ACKNOWLEDGMENTS}

The authors are grateful to the National Science Foundation and the American Chemical Society for financial support. We are also grateful to Xerox, Dupont, and 3M for their support.

\section{APPENDIX A: ECCB FORMULAS}

The formulas presented here provide the upper limit distance $d_{\max }$ required by the ECCB scheme outlined in Sec. II [Eq. (3)]. We consider the case of chains with equal-size spherical sites of diameter $\sigma=1$. The bond length is $l$ and the formulas are valid for $\sqrt{3} / 3 \geqslant l / \sigma \geqslant 1$. Let sites 0 and $(n+1)$ be the open sites at a given step in the regrowing process; the 
distance between these two sites is $d_{0}$. We are trying to regrow site 1 (sites $1,2, \ldots, n$ remain to be regrown). Let us define the limiting distances

$$
\begin{aligned}
& d_{b_{j}}^{2}=1+(j-1) j l^{2} \quad j=1,2, \ldots, n-1, \\
& d_{b_{n}}^{2}=n+[(n-1) l]^{2}
\end{aligned}
$$

and the characteristic angles,

$$
\begin{aligned}
& \theta_{1}=\arccos \left(\frac{1}{2 l}\right), \\
& \theta_{2}=\arccos \left(1-\frac{l^{2}}{2}\right), \\
& \phi_{j}=\theta_{1}+j \theta_{2} \quad j=0,1, \ldots, n-1 . \\
& \text { If } d_{0}^{2}>d_{b_{n}}^{2} \text { then } \\
& d_{\max }^{2}=(n l)^{2},
\end{aligned}
$$

otherwise

$$
d_{\max }^{2}=l^{2}+d_{0}^{2}-2 l d_{0} \cos \theta,
$$

where

$$
\begin{aligned}
& \theta=\min \left[\pi, \theta^{*}+\phi_{n-1-j}\right], \\
& \theta^{*}=\arccos \left\{\frac{1}{2 d_{0}}\left[1+d_{0}^{2}-(j l)^{2}\right]\right\}
\end{aligned}
$$

the value of $j$ to be used in the last two equations identifies the first distance $d_{b_{j}}$ for which the condition $d_{b_{j}}<d_{0}$ is satisfied when going in decreasing order from $j=n-1$ to $j=1$. The angle $\theta$ gives the angle between the growing bond and vector $\mathbf{d}_{0}$ when the maximal extension of the chain portion between fixed sites 0 and $n+1$ is reached. ${ }^{14}$

\section{APPENDIX B: FORMULAS FOR TRIPODE MOVE}

Let sites 0,2 , and 3 be the neighbors of site 1 , the node to be repositioned. The two positions that site 1 can take can be derived by geometric considerations in various ways. Here, we present one method based on the local coordinate system given in Fig. 14. The $z$ axis lies along the line $\overline{02}$ and the $x$ axis lies on the plane defined by sites 0,2 , and 3. Point $q$, the center of the coordinate system, is the center of rotation of site 1 around line $\overline{02}$. Point $c$ is the projection of site 3 on $\overline{02}$. If $b_{i j}$ and $d_{i j}$ denote the bond length and the distance between points $i$ and $j$, respectively (depending on whether or not such sites are neighbors), we can write

$$
\begin{aligned}
& d_{0 c}=\frac{1}{2 d_{02}}\left(d_{03}^{2}+d_{02}^{2}-d_{23}^{2}\right), \\
& d_{0 q}=\frac{1}{2 d_{02}}\left(b_{01}^{2}+d_{02}^{2}-b_{12}^{2}\right) .
\end{aligned}
$$

Points $c$ and $q$ are then given by

$$
\mathbf{X}_{c}=\mathbf{X}_{0}+\frac{d_{0 c}}{d_{02}}\left(\mathbf{X}_{2}-\mathbf{X}_{0}\right),
$$

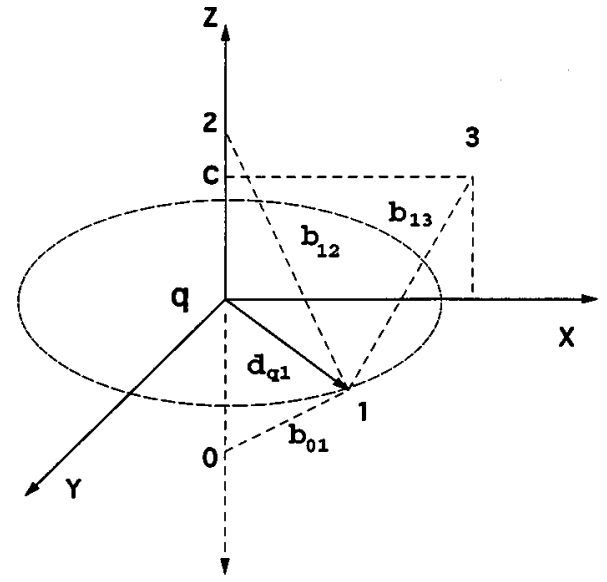

FIG. 14. Local coordinate system for a TRIPODE move. Site 1 is the node and sites 0,2 , and 3 are the neighbor sites to which site 1 needs to be attached. Sites 0,2 , and 3 form the $x-z$ plane.

$$
\mathbf{X}_{q}=\mathbf{X}_{0}+\frac{d_{0 q}}{d_{02}}\left(\mathbf{X}_{2}-\mathbf{X}_{0}\right)
$$

where $\mathbf{X}$ denotes the coordinates of the subindexed point. The new position of site 1 is given by

$$
\mathbf{X}_{1}=\mathbf{X}_{q}+d_{q 1} \cos \phi \mathbf{u}+d_{q 1} \sin \phi \mathbf{v},
$$

where

$$
\phi=\arccos \left\{\frac{1}{2 d_{c 3} d_{q 1}}\left[d_{q 3}^{2}+d_{q 1}^{2}-b_{13}^{2}\right]\right\}
$$

and $\mathbf{u}$ and $\mathbf{v}$ are unit vectors along the temporary $x$ and $y$ axis, respectively. These vectors can be computed from

$$
\begin{aligned}
& \mathbf{u}=\frac{1}{d_{c 3}}\left(\mathbf{X}_{3}-\mathbf{X}_{c}\right), \\
& \mathbf{v}=\frac{1}{d_{02} d_{c 3}}\left[\left(\mathbf{X}_{2}-\mathbf{X}_{0}\right) \times\left(\mathbf{X}_{3}-\mathbf{X}_{0}\right)\right] .
\end{aligned}
$$

The two possible positions of site 1 are obtained by letting angle $\phi$ be less than $\pi$ or greater than $\pi$, respectively.

\section{APPENDIX C: JACOBIAN DETERMINANTS FOR BRANCHED CHAINS}

The Jacobian determinant arises because the ECCB moves adopted in our work to reposition sites actually use different degrees of freedom than those used by an unconstrained growing process. In an unconstrained regrowing process, the orientation of the growing bond is determined by random selection of a random angle $\phi$ in $[-\pi, \pi]$ and a latitude $\cos \theta$ in $[-1,1]$ (spherical coordinates). Angle $\phi$ is chosen around a circumference centered at the previous site, and $\theta$ is the angle between the growing vector and the vector normal to the $\phi$ circle. In principle, the reference axis to position these angles is arbitrary. In the following discussion, we label these angles with a subindex that identifies the growing site (i.e., the end point of the growing vector).

The determinant Jacobian associated with the repositioning of bifunctional sites by an ISOPLANE move is given in 
Ref. 15. The determinant Jacobian was found to be analogous to that of a ECCB move using mode 1 sampling. If $i$ denotes the dangling site for the ISOPLANE move, or the last site in an ECCB move, we then have

$$
\mathbf{J}=\frac{1}{b_{i-1, i} b_{i, i+1} d_{i-1, i+1}},
$$

where $b_{j, k}$ and $d_{j, k}$ denote the bond length and the actual distance between sites $j$ and $k$, respectively.

When regrowing a chain portion which may include nodes, we can separate this process into steps. The first step begins with the first growing site and ends with the first node found in the path (including such node), the second step then includes the following sites until the next node is reached, and so on until closing the chain. The transformation of degrees of freedom associated with the overall process is compounded by the corresponding transformations occurring at each step. More precisely, the Jacobian determinant of the total process is given by the product of the Jacobian determinants of the $N_{\text {steps }}$ steps,

$$
\mathbf{J}=\prod_{i=1}^{N_{\text {steps }}} \mathbf{J}_{i}
$$

The reason that transformations can be evaluated independently at each step is that under mode 1 sampling, there are no geometric constraints relating sites in one step section and sites yet to be grown in the next steps. Likewise, no restrictions of degrees of freedom are inherited from step to step.

We now describe in more detail the evaluation of the
Jacobian determinant associated with the repositioning of trifunctional and tetra-functional sites.

\section{Nodes grown as bifunctional sites}

Depending on the growing scheme of a particular chain portion, a tri- or tetra-functional node can actually be grown as a bifunctional site. For instance if two of the neighbor sites of a tetra-functional node remain to be regrown, then the node needs to be attached to the other two neighbors only (those already present). Let us use Fig. 1 to illustrate a growing process where a tri-functional node (site 1) is actually grown as a bifunctional site. Let us assume that at some point in the growing process, sites $2^{\prime \prime}, 1$, and 2 remain to be grown, in that order. The change in degrees of freedom can be determined by interpreting the growing process as one in which sites $2^{\prime \prime}, 1,2,3$, and $2^{\prime}$ are grown. In the constrained (actual) process, the positions of sites $2^{\prime}$ and 3 are fixed, e.g.,

$$
\begin{aligned}
& \phi_{2^{\prime \prime}}, \cos \theta_{2^{\prime \prime}}, \phi_{1}, \cos \theta_{1}, \phi_{2^{\prime}}, \cos \theta_{2^{\prime}}, \phi_{2}, \cos \theta_{2}, \phi_{3}, \cos \theta_{3} \\
& \quad \rightarrow \phi_{2^{\prime \prime}}, \cos \theta_{2^{\prime \prime}}, \phi_{1}, \cos \theta_{1}, \mathbf{r}_{2^{\prime}}, \phi_{2}, \mathbf{r}_{3} .
\end{aligned}
$$

Since mode 1 sampling is being used throughout this work, ${ }^{14}$ the common degrees of freedom in the transformation can be removed and the volume elements in the two coordinate frames are related by,

$$
\begin{aligned}
& d \phi_{2^{\prime}} d\left(\cos \theta_{2^{\prime}}\right) d\left(\cos \theta_{1}\right) d\left(\cos \theta_{2}\right) d \phi_{3} d\left(\cos \theta_{3}\right) \\
& \quad \rightarrow \mathbf{J} d \mathbf{r}_{2^{\prime}} d \mathbf{r}_{3},
\end{aligned}
$$

where

$$
\mathbf{J}^{-1}=\left|\begin{array}{cccccc}
\frac{\partial \mathbf{r}_{2^{\prime}}}{\partial \phi_{2^{\prime}}} & \frac{\partial \mathbf{r}_{2^{\prime}}}{\partial \cos \theta_{2^{\prime}}} & \frac{\partial \mathbf{r}_{2^{\prime}}}{\partial \cos \theta_{1}} & \frac{\partial \mathbf{r}_{2^{\prime}}}{\partial \cos \theta_{2}} & \frac{\partial \mathbf{r}_{2^{\prime}}}{\partial \phi_{3}} & \frac{\partial \mathbf{r}_{2^{\prime}}}{\partial \cos \theta_{3}} \\
\frac{\partial \mathbf{r}_{3}}{\partial \phi_{2^{\prime}}} & \frac{\partial \mathbf{r}_{3}}{\partial \cos \theta_{2^{\prime}}} & \frac{\partial \mathbf{r}_{3}}{\partial \cos \theta_{1}} & \frac{\partial \mathbf{r}_{3}}{\partial \cos \theta_{2}} & \frac{\partial \mathbf{r}_{3}}{\partial \phi_{3}} & \frac{\partial \mathbf{r}_{3}}{\partial \cos \theta_{3}}
\end{array}\right|
$$

This $6 \times 6$ matrix can be split into 4 smaller $3 \times 3$ sections (keeping the order of all entries) as follows,

$$
\mathbf{J}^{-1}=\left|\begin{array}{cc}
B & C \\
D & E
\end{array}\right| .
$$

It is easy to show that matrix $C$ has all zero entries; we can therefore write

$$
\mathbf{J}^{-1}=|E|\left|\left(B-C E^{-1} D\right)\right|=|E||B|,
$$

where $|B|^{-1}$ and $|E|^{-1}$ are the Jacobian determinants associated with the repositioning of sites 1 and 2, respectively (see Ref. 14). This result is in agreement with Eq. (C2), i.e., the determinants $|B|^{-1}$ and $|E|^{-1}$ are associated with two steps: growth of sites $2^{\prime \prime}$ and 1 (step 1), and then growth of site 2 (step 2), respectively. Note that determinant $|B|^{-1}$ reflects the fact that site 1 is appended in a crank-shaft fashion, i.e., as a bifunctional site. A similar analysis can be applied to any configuration involving tri-functional or $n$-functional nodes provided that in the growing process they act as bifunctional sites.

\section{Nodes grown as tri-functional sites}

For simplicity let us first analyze the repositioning of a node. This case corresponds to a TRIPODE move. Figure 15 illustrates the coordinate system to be used. The node to be grown is site 1 . The $z$ axis lies along the $\overline{02}$ line, and the $x$ axis lies on the plane formed by the centers of sites 0,2 , and 3 . The unconstrained growing process is assumed to begin with site 1 and then proceed to sites 2 and 3 . In the constrained process the positions of sites 2 and 3 are fixed, the transformation of degrees of freedom is then given by

$$
\phi_{1}, \cos \theta_{1}, \phi_{2}, \cos \theta_{2}, \phi_{3}, \cos \theta_{3} \rightarrow \mathbf{r}_{2}, \mathbf{r}_{3}
$$

and the two volume elements are related by 


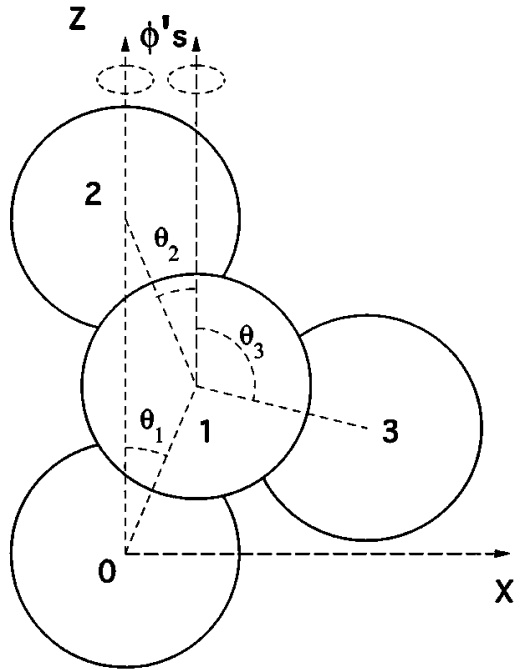

FIG. 15. Local configuration of sites around the nodal site 1 . Sites 0 , 2, and 3 form the $x-z$ plane and line $\overline{02}$ gives the $z$ direction. The $\theta$ angles are formed between the connecting bond vector and the $z$ axis while the $\phi$ angles give the rotation of such vector from the $x$ axis around the $z$ axis.

$d \phi_{1} d\left(\cos \theta_{1}\right) d \phi_{2} d\left(\cos \theta_{2}\right) d \phi_{3} d\left(\cos \theta_{3}\right)$

$$
=\mathbf{J} d \mathbf{r}_{2} d \mathbf{r}_{3},
$$

where

$$
\begin{aligned}
& \mathbf{J}=\frac{\sin \theta_{1} \sin \theta_{2} \sin \theta_{3}}{|A|}, \\
& |A|=\left|\begin{array}{llllll}
\frac{\partial \mathbf{r}_{2}}{\partial \phi_{1}} & \frac{\partial \mathbf{r}_{2}}{\partial \theta_{1}} & \frac{\partial \mathbf{r}_{2}}{\partial \phi_{2}} & \frac{\partial \mathbf{r}_{2}}{\partial \theta_{2}} & \frac{\partial \mathbf{r}_{2}}{\partial \phi_{3}} & \frac{\partial \mathbf{r}_{2}}{\partial \theta_{3}} \\
\frac{\partial \mathbf{r}_{3}}{\partial \phi_{1}} & \frac{\partial \mathbf{r}_{3}}{\partial \theta_{1}} & \frac{\partial \mathbf{r}_{3}}{\partial \phi_{2}} & \frac{\partial \mathbf{r}_{3}}{\partial \theta_{2}} & \frac{\partial \mathbf{r}_{3}}{\partial \phi_{3}} & \frac{\partial \mathbf{r}_{3}}{\partial \theta_{3}}
\end{array}\right| .
\end{aligned}
$$

The positions of sites 2 and 3 are given by

$$
\begin{aligned}
\mathbf{r}_{k}= & \mathbf{r}_{0}+b_{01}\left[\sin \theta_{1} \cos \phi_{1} \mathbf{u}_{x}+\sin \theta_{1} \sin \phi_{1} \mathbf{u}_{y}\right. \\
& \left.+\cos \theta_{1} \mathbf{u}_{z}\right]+b_{1 k}\left[\sin \theta_{k} \cos \phi_{k} \mathbf{u}_{x}\right. \\
& \left.+\sin \theta_{k} \sin \phi_{k} \mathbf{u}_{y}+\cos \theta_{k} \mathbf{u}_{z}\right] \quad k=2,3,
\end{aligned}
$$

where $b_{i j}$ is the length of the bond joining sites $i$ and $j$ and where the u's denote unit vectors in the direction of the subindexed axis. by

The partial derivatives required to evaluate $|A|$ are given

$$
\frac{\partial \mathbf{r}_{k}}{\partial \phi_{1}}=b_{01} \sin \theta_{1}\left[-\sin \phi_{1} \mathbf{u}_{x}+\cos \phi_{1} \mathbf{u}_{y}\right]
$$

$\frac{\partial \mathbf{r}_{k}}{\partial \theta_{1}}=b_{01}\left[\cos \theta_{1} \cos \phi_{1} \mathbf{u}_{x}+\cos \theta_{1} \sin \phi_{1} \mathbf{u}_{y}-\sin \theta_{1} \mathbf{u}_{z}\right]$,

$$
\frac{\partial \mathbf{r}_{k}}{\partial \phi_{k}}=b_{1 k} \sin \theta_{k}\left[-\sin \phi_{k} \mathbf{u}_{x}+\cos \phi_{k} \mathbf{u}_{y}\right]
$$

$\frac{\partial \mathbf{r}_{k}}{\partial \theta_{k}}=b_{1 k}\left[\cos \theta_{k} \cos \phi_{k} \mathbf{u}_{x}+\cos \theta_{k} \sin \phi_{k} \mathbf{u}_{y}-\sin \theta_{k} \mathbf{u}_{z}\right]$,

in all the equations above, $k=2,3$. Using these equations, the Jacobian determinant now takes the form
This determinant can be evaluated analytically and further reduced by taking into account the fact that by construction $\phi_{2}=\phi_{1} \pm \pi$; a very simple final expression for the Jacobian determinant is then derived

$$
\begin{aligned}
\mathbf{J}^{-1} & =b_{01}^{2} b_{12}^{2} b_{13}^{2} \sin \left(\theta_{1}+\theta_{2}\right) \sin \theta_{3} \sin \left(\phi_{3}-\phi_{1}\right) \\
& =b_{01} b_{12} b_{13} d_{02} d_{c 3} d_{q 1} \sin \phi_{1},
\end{aligned}
$$

where the meaning of the symbols $d_{i j}$ is consistent with the description given in Appendix B.

The Jacobian determinant associated with the repositioning of a tetra-functional node by growing it as a trifunctional node (i.e., a TRIPODE move) can be evaluated by resorting to Eq. (C2). An illustrative example is presented next. Figure 16 shows a possible configuration. The single growing path is formed by sites 0,1 , and 4 . This time the implied transformation is 


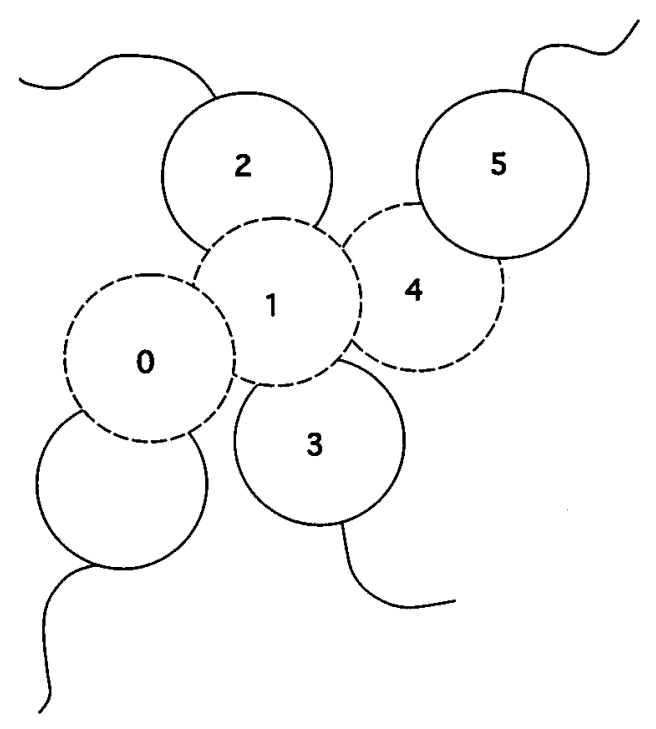

FIG. 16. Local configuration of a chain portion around a tetra-functional node (site 1). Sites with dashed contour are the sites to be repositioned. The definition of $\theta$ and $\phi$ angles follows the same rules as those illustrated in Fig. 14.

$$
\begin{gathered}
\phi_{0}, \cos \theta_{0}, \phi_{1}, \cos \theta_{1}, \phi_{2}, \cos \theta_{2}, \phi_{3}, \cos \theta_{3}, \phi_{4} \\
\cos \theta_{4}, \phi_{5}, \cos \theta_{5} \rightarrow \phi_{0}, \cos \theta_{0}, \mathbf{r}_{2}, \mathbf{r}_{3}, \mathbf{r}_{5}, \phi_{4}
\end{gathered}
$$

After removing common degrees of freedom, the Jacobian determinant can be expressed as

$$
\mathbf{J}^{-1}=\frac{1}{\sin \theta_{1} \sin \theta_{2} \sin \theta_{3} \sin \theta_{4} \sin \theta_{5}}\left|\begin{array}{ll}
A & B \\
C & D
\end{array}\right|,
$$

where the determinant of $A$ is exactly that of Eq. (C3) and where

$$
\begin{aligned}
& B=\left|\begin{array}{lll}
\frac{\partial \mathbf{r}_{2}}{\partial \phi_{4}} & \frac{\partial \mathbf{r}_{2}}{\partial \phi_{5}} & \frac{\partial \mathbf{r}_{2}}{\partial \theta_{5}} \\
\frac{\partial \mathbf{r}_{3}}{\partial \phi_{4}} & \frac{\partial \mathbf{r}_{3}}{\partial \phi_{5}} & \frac{\partial \mathbf{r}_{3}}{\partial \theta_{5}}
\end{array}\right|
\end{aligned}
$$

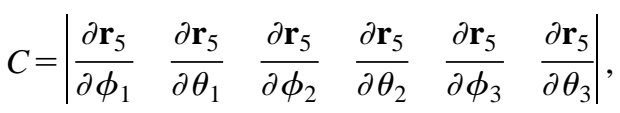

$$
\begin{aligned}
& D=\left|\begin{array}{lll}
\frac{\partial \mathbf{r}_{5}}{\partial \phi_{4}} & \frac{\partial \mathbf{r}_{5}}{\partial \phi_{5}} & \frac{\partial \mathbf{r}_{5}}{\partial \theta_{5}}
\end{array}\right| .
\end{aligned}
$$

Noting that matrix $B$ is a null matrix, we arrive at

$$
\begin{aligned}
\mathbf{J} & =\frac{\sin \theta_{1} \sin \theta_{2} \sin \theta_{3} \sin \theta_{4} \sin \theta_{5}}{|A|\left|D-B A^{-1} C\right|} \\
& =\left[\frac{\sin \theta_{1} \sin \theta_{2} \sin \theta_{3}}{|A|}\right]\left[\frac{\sin \theta_{4} \sin \theta_{5}}{|D|}\right] \\
& =\mathbf{J}_{\mathrm{I}} \mathbf{J}_{\mathrm{II}},
\end{aligned}
$$

where $\mathbf{J}_{\mathrm{I}}$ is immediately identified as the Jacobian determinant of a TRIPODE move of site 1 around sites 0, 2, and 3, just as in our previous example, while $\mathbf{J}_{\mathrm{II}}$ is the Jacobian determinant corresponding to a crank-shaft move of site 4 around sites 1 and 5 (which for slab volume moves would be an ISOPLANE move). Once again, this result is in agreement with the general rule, Eq. (C2).

${ }^{1}$ S. Duane, A. D. Kennedy, B. J. Pendelton, and D. Roweth, Phys. Lett. B 195, 216 (1987); B. M. Forrest and U. W. Suter, J. Chem. Phys. 101, 2616 (1994).

${ }^{2}$ D. C. Rapaport, J. Chem. Phys. 71, 3299 (1979).

${ }^{3}$ A. Bellemans, J. Orban, and D. Van Belle, Mol. Phys. 39, 781 (1980).

${ }^{4}$ N. Metropolis, A. W. Rosenbluth, M. N. Rosenbluth, A. H. Tell, and E. Teller, J. Chem. Phys. 21, 1087 (1953).

${ }^{5}$ M. P. Allen and D. J. Tildesley, Computer Simulation of Liquids (Clarendon, Oxford, 1987).

${ }^{6}$ F. T. Wall and F. Mandel, J. Chem. Phys. 63, 4592 (1975); R. H. Boyd, Macromolecules 22, 2477 (1989).

${ }^{7}$ A. Yethiraj and C. K. Hall, J. Chem. Phys. 94, 3943 (1991).

${ }^{8}$ J. Gao and J. H. Weiner, Macromolecules 21, 773 (1988); J. H. Weiner and J. Gao, ibid. 23, 1860 (1990).

${ }^{9}$ G. S. Grest, K. Kremer, and E. R. Duering, Europhys. Lett. 19, 195 (1992).

${ }^{10}$ G. S. Grest, K. Kremer, and E. R. Duering, Physica A 194, 330 (1993).

${ }^{11}$ E. R. Duering, K. Kremer, and G. S. Grest, J. Chem. Phys. 101, 8169 (1994).

${ }^{12}$ J. J. de Pablo, M. Laso, and U. W. Suter, J. Chem. Phys. 96, 2395 (1992).

${ }^{13}$ D. Frenkel, G. C. A. M. Mooij, and B. Smit, J. Phys. 4, 3053 (1992).

${ }^{14}$ F. A. Escobedo and J. J. de Pablo, J. Chem. Phys. 102, 2636 (1995).

${ }^{15}$ F. A. Escobedo and J. J. de Pablo, Macromol. Chem. Phys. Theory Simulation 4, 691 (1995).

${ }^{16}$ F. A. Escobedo and J. J. de Pablo, Mol. Phys. (in press).

${ }^{17}$ S. Phan, E. Kierlik, M. L. Rosinberg, H. Yu, and G. Stell, J. Chem. Phys. 29, 5326 (1993).

${ }^{18}$ F. A. Escobedo and J. J. de Pablo, J. Chem. Phys. 103, 1946 (1995).

${ }^{19}$ D. Moller and J. Fischer, Mol. Phys. 69, 463 (1990).

${ }^{20} \mathrm{R}$. Everaers and K. Kremer, Macromolecules (submitted).

${ }^{21}$ R. Everaers and K. Kremer Phys. Rev. Lett. (submitted).

${ }^{22}$ J. Chang and S. I. Sandler, Chem. Eng. Sci. 49, 2777 (1994).

${ }^{23}$ A. Yethiraj and C. K. Hall, J. Chem. Phys. 95, 1999 (1991). A. Yethiraj and C. K. Hall, ibid. 95, 8494 (1991).

${ }^{24}$ J. J. de Pablo, J. M. Prausnitz, H. J. Strauch, and P. T. Cummings, J. Chem. Phys. 93, 209 (1990).

${ }^{25}$ F. W. Billmeyer, Jr., Textbook of Polymer Science, 3rd ed. (Wiley, New York, 1984)

${ }^{26}$ J. M. Prausnitz, R. N. Lichtenthaler, and E. Gomes de Azevedo, Molecular Thermodynamics of Fluid-phase Equilibria, 2nd ed. (Prentice-Hall, New York, 1986).

${ }^{27}$ P. J. Flory, Principles of Polymer Chemistry (Cornell University, New York, 1953). 